\title{
Analysis of NMR Wide Line Shapes Generated by Two Broadening Mechanisms
}

\author{
S. E. S V A N S O N \\ Research Group for NMR, Division of Physical Chemistry, The Royal Institute of Technology, \\ Stockholm 70, Sweden
}

Dedicated to Professor Ole Lamm on his 60th birthday

\begin{abstract}
The use of Fourier transforms for the analysis of NMR wide line spectra generated by two broadening mechanisms is discussed. Some examples are given of analysis performed for spectra perturbed by quadrupole interaction and also of spectra of crystalline solids containing groups of interacting nuclei.
\end{abstract}

$T^{2}$

The line-shape of NMR wide line spectra is, in many cases, determined by two or more broadening mechanisms. The most important factors responsible for the line-shape of this type of spectra are the nuclear dipole-dipole interaction and the magnetic and electric nuclear-electronic interactions. The dipole-dipole interaction is always of importance in non-rotating specimens ${ }^{1}$ and must be taken into account in all cases where any other broadening mechanism is present.

As an example of a spectral type generated by two broadening mechanisms, the NMR spectra perturbed by quadrupole couplings in polycrystalline samples, may be studied. The following functional assignments are assumed for the generation of the modified line-shape. Let $Q(\xi)$ denote the line-shape yielded by the quadrupole coupling. The fraction of nuclei $Q(\xi) \xi$ with a displacement in the interval $\xi$ to $\xi+d \xi$ is assumed to be distributed over the function $K(x, \xi)$ due to the dipole-dipole interaction. $K$ is, moreover, subjected to the condition

$$
\int_{-\infty}^{+\infty} K(x, \xi) \mathrm{d} x,=1
$$

The absorption line may be written

$$
f(x)=\int_{\xi_{1}}^{\xi_{2}} K(x, \xi) Q(\xi) \mathrm{d} \xi
$$


where the function $Q(\xi)$ is defined in the interval $\xi_{1}$ to $\xi_{2}$. If the two broadening mechanisms are mutually independent, the function due to the dipoledipole interaction can be written as $P(x-\xi)$, and the absorption line takes the form

$$
f(x)=\int_{\xi_{1}}^{\xi_{2}} P(x-\xi) Q(\xi) \mathrm{d} \xi
$$

This equation is given earlier, for example by Hughes and Mac Donald ${ }^{2}$. In mathematical texts $f(x)$ in eqn. (2) is called the convolution of $P$ and $Q$. The theory of the dipole-dipole interaction shows that the $P$-function is symmetric, which is utilized in the following to simplify the analysis. It should be remembered however that individual dipole-broadened component lines in a spectrum are not always symmetric as has been shown by Itoh $e t a l .^{3}$ and Silver et $a l .{ }^{4}$, and in such cases the following deductions are not applicable.

With these assumptions, the nth moment of $f(x)$ is easily calculated. In particular

$$
\begin{aligned}
M_{\mathrm{o}}=\int_{-\infty}^{+\infty} f(x) \mathrm{d} x & =\int_{-\infty}^{+\infty} \int_{\xi_{1}}^{\xi_{2}} P(x-\xi) Q(\xi) \mathrm{d} \xi \mathrm{d} x=\int_{\xi_{1}}^{\xi_{2}} Q(\xi)\left\{\int_{-\infty}^{+\infty} P(x-\xi) \mathrm{d} x\right\} \mathrm{d} \xi \\
& =\int_{\xi_{1}}^{\xi_{2}} Q(\xi)\left\{\int_{-\infty}^{+\infty} P(\zeta) \mathrm{d} \zeta\right\} \mathrm{d} \xi=\int_{\xi_{1}}^{\xi_{1}} Q(\xi) \mathrm{d} \xi \\
M_{1} & \left.=\int_{-\infty}^{+\infty} x \cdot f(x) \mathrm{d} x=\int_{\xi_{1}}^{\xi_{2}} Q(\xi)\left\{\int_{-\infty}^{+\infty} x P(x-\xi) \mathrm{d} x\right)\right\} \mathrm{d} \xi= \\
& =\int_{\xi_{1}}^{\xi_{2}} Q(\xi)\left\{\int_{-\infty}^{+\infty}(\zeta+\xi) P(\zeta) \mathrm{d} \zeta\right\} \mathrm{d} \xi=\int_{\xi_{1}}^{\xi_{2}} \xi Q(\xi) \mathrm{d} \xi
\end{aligned}
$$

as $\int_{-\infty}^{+\infty} \zeta \cdot P(\zeta) \mathrm{d} \zeta=0$ for even functions $P(\zeta)$.

$M_{\mathrm{o}}$ and $M_{1}$ are thus independent of $P$. This well-known fact has, for example, been used by Otsuka and Kawamura ${ }^{5}$ in a study of dislocation effects on the iodine-resonance in some crystalline alkali iodides. All higher moments depend on $P$. So is for example

$$
M_{2}=\int_{\xi_{1}}^{\xi_{2}} \xi^{2} \cdot Q(\xi) \mathrm{d} \xi+\int_{-\infty}^{+\infty} \zeta^{2} \cdot P(\zeta) \mathrm{d} \zeta \cdot \int_{\xi_{1}}^{\xi_{2}} Q(\xi) \mathrm{d} \xi
$$

The simple analytical form of $M_{1}$ is due to the assumed symmetry of the function $P(\zeta)$. Similarly, the sine and cosine transforms of $f(x)$ lead to very simple analytical expressions if $P(\zeta)$ is assumed to be even, since in this case the integral

$$
\int_{-\infty}^{+\infty} P(\zeta) \cdot \sin (u \zeta) \mathrm{d} \zeta
$$

Acta Chem. Scand. 16 (1962) No. 9 
disappears, and

$$
\begin{array}{r}
F(u)=\int_{-\infty}^{+\infty} f(x) \cdot \cos (u x) \mathrm{d} x=\int_{-\infty}^{+\infty} \int_{\xi 1}^{\xi_{2}} P(\zeta) \cdot \cos u(\zeta+\xi) \mathrm{d} \zeta \cdot Q(\xi) \mathrm{d} \xi= \\
=\int_{-\infty}^{+\infty} P(\zeta) \cdot \cos (u \zeta) \mathrm{d} \zeta \cdot \int_{\xi_{1}}^{\xi_{2}} Q(\xi) \cdot \cos (u \xi) \mathrm{d} \xi \\
G(u)=\int_{-\infty}^{+\infty} f(x) \cdot \sin (u x) \mathrm{d} x=\int_{-\infty}^{+\infty} \int_{\xi_{1}}^{\xi_{2}} P(\zeta) \cdot \sin u(\zeta+\xi) \mathrm{d} \zeta \cdot Q(\xi) \mathrm{d} \xi= \\
=\int_{-\infty}^{+\infty} P(\zeta) \cdot \cos (u \zeta) \mathrm{d} \zeta \cdot \int_{\xi_{1}}^{\xi_{2}} Q(\xi) \cdot \sin (u \xi) \mathrm{d} \xi
\end{array}
$$

Relations of this kind are well-known from the theory of the convolution. From eqns. (3) and (4) we have

$$
\frac{F(u)}{G(u)}=\frac{\int_{\xi_{1}}^{\xi_{2}} Q(\xi) \cdot \cos (u \xi) \mathrm{d} \xi}{\int_{\xi_{1}}^{\xi_{2}} Q(\xi) \cdot \sin (u \xi) \mathrm{d} \xi}
$$

With this expression it is possible to make an assumed analytical form of $Q(\xi)$ with unknown parameters to coincide with experimental data, without making any other assumptions than those stated above about the function $P(\zeta)$. The development can be made in a large interval of $u$-values.

The method can be simplified for practical use by calculating the Fouriertransforms of the function $f^{\prime}(x)$, measured in the wide line technique.

Here is

and we compute

$$
f^{\prime}(x)=\int_{\xi_{1}}^{\xi_{2}} P^{\prime}(x-\xi) Q(\xi) \mathrm{d} \xi
$$

$$
\begin{aligned}
& F_{1}(u)=\int_{-\infty}^{+\infty} f^{\prime}(x) \cdot \cos (u x) \mathrm{d} x=-\int_{-\infty}^{+\infty} P^{\prime}(\zeta) \cdot \sin (u \zeta) \mathrm{d} \zeta \cdot \int_{\xi_{1}}^{\xi_{2}} Q(\xi) \cdot \sin (u \xi) \mathrm{d} \xi \\
& G_{1}(u)=\int_{-\infty}^{+\infty} f^{\prime}(x) \cdot \sin (u x) \mathrm{d} x=\int_{-\infty}^{+\infty} P^{\prime}(\zeta) \cdot \sin (u \zeta) \mathrm{d} \zeta \cdot \int_{\xi_{1}}^{\xi_{2}} Q(\xi) \cdot \cos (u \xi) \mathrm{d} \xi
\end{aligned}
$$

$\operatorname{as} \int_{-\infty}^{+\infty} P^{\prime}(\zeta) \cos (u \zeta) \mathrm{d} \zeta=0$ for odd functions $P^{\prime}(\zeta)$

The integral

$$
\int_{-\infty}^{+\infty} P^{\prime}(\zeta) \cdot \sin (u \zeta) \mathrm{d} \zeta=\int_{-\infty}^{+\infty} P(\zeta) \cdot \sin (u \zeta)-u \int_{-\infty}^{+\infty} P(\zeta) \cdot \cos (u \zeta) \mathrm{d} \zeta=
$$




$$
=-u \int_{-\infty}^{+\infty} P(\zeta) \cdot \cos (u \zeta) \mathrm{d} \zeta
$$

as $P(\zeta)$ goes towards zero in both limits.

Thus eqn. (3) can be substituted by

$$
\frac{F_{1}(u)}{u}=\int_{-\infty}^{+\infty} P(\zeta) \cdot \cos (u \zeta) \mathrm{d} \zeta \cdot \int_{\xi_{1}}^{\xi_{2}} Q(\xi) \cdot \sin (u \xi) \mathrm{d} \xi
$$

and eqn. (4) by

$$
-\frac{G_{1}(u)}{u}=\int_{-\infty}^{+\infty} P(\zeta) \cdot \cos (u \zeta) \mathrm{d} \zeta \cdot \int_{\xi_{1}}^{\xi_{2}} Q(\xi) \cdot \cos (u \xi) \mathrm{d} \xi
$$

Finally eqn. (5) can be replaced by

$$
-\frac{G_{1}(u)}{F_{1}(u)}=\frac{\int_{\xi_{1}}^{\xi_{2}} Q(\xi) \cdot \cos (u \xi) \mathrm{d} \xi}{\int_{\xi_{1}}^{\xi_{2}} Q(\xi) \cdot \sin (u \xi) \mathrm{d} \xi}
$$

In the following eqns. (3), (4), and (5) have been used but it is assumed, however, that $F(u), G(u)$ and $F(u) / G(u)$ are replaced by $-G_{1}(u) / u, F_{1}(u) / u$ and $-G_{1}(u) / F_{1}(u)$, respectively, for reasons of convenience.

This transformation is of great practical importance in the numerical calculations since the integrals $F_{1}(u)$ and $G_{1}(u)$ converge faster than $F(u)$ and $G(u)$. For small values of $u$ the convergence is the same as for $\int_{-\infty}^{+\infty} f^{\prime}(x) \mathrm{d} x$, and for large values of $u$ the convergence is still faster due to the alternating signs of the successive contributions in both limits.

When the constant $u$ is large, the evaluation of the integrals becomes difficult because of the violent oscillation of the trigonometric functions. A method for the evaluation of the integrals in this case is given by Kopal ${ }^{6}$ yielding correct results and error estimates for a limited number of measured points on the curve.

Also, if the function $Q(\xi)$ is symmetric, the eqn. (4) disappears. The use of eqn. (5) is consequently limited to unsymmetrical functions $Q(\xi)$. Such functions appear in the case of polycrystalline samples subjected to secondorder quadrupole interaction or anisotropic Knight shift.

The displacement due to the quadrupole interaction, first- or secondorder, depends on the orientation of the external magnetic field in relation to the principal axes of the electric field gradient tensor. The condition of mutual independence of the distributions $P(\zeta)$ and $Q(\xi)$ will thus in this case require that the function $P(\zeta)$ describing the dipolar broadening is independent of the orientation of the external magnetic field in the crystal lattice. This

Acta Chem. Scand. 16 (1962) No. 9 
requirement of isotropy of $P(\zeta)$ is indispensable for a detailed analysis of all spectra of polycrystalline samples, and it seems hardly possible ever to resolve spectra of this kind, unless this condition is fulfilled.

The aforementioned investigation by Otsuka and Kawamura provides an example of a single crystal giving rise to an unsymmetric function $Q(\xi)$ of interest for the future study of this problem and the applicability of eqn. (5).

Many examples of symmetric functions $Q(\xi)$ exist. Valuable information can in this case be obtained from eqn. (3) if the integral $\int_{\xi_{1}}^{\xi_{2}} Q(\xi) \cdot \cos (u \xi) \mathrm{d} \xi$ has zeros that can be distinguished from the zeros of $\int_{-\infty}^{+\infty} P(\zeta) \cdot \cos (u \zeta) \mathrm{d} \zeta$.

Assuming $P(\zeta)$ to be Gaussian, $P(\zeta)=\mathrm{e}^{-a \zeta^{2}}$, the cosine transform is $+\infty$

$\int_{-\infty} P(\zeta) \cdot \cos (u \zeta) \mathrm{d} \zeta=\sqrt{\frac{\pi}{\alpha}} \cdot \mathrm{e}^{-u^{2} / 4 \alpha}$. A narrow function $P(\zeta)$ thus has a broad transform and vice versa.

The experimental $P(\zeta)$ curves of pure dipole-broadened functions give transforms which, for small $u$, coincide rather well with the Gaussian and go towards zero as $u$ goes towards infinity. For large $u$, however, the transforms usually have a sequence of zeros, and the narrower the dipole-broadened function the higher the $u$-values for the zeros.

When $P(\zeta)$ is narrow enough in comparison with the $Q(\xi)$-function, the zeros in the transform of $Q(\xi)$ will occur for smaller $u$-values than the zeros in the transform of $P(\zeta)$. In this case, the zeros are easily distinguished.

Polycrystalline samples perturbed by first-order quadrupole interaction, in all cases lead to symmetric functions $Q(\xi)$.

Spectra of polycrystalline solids where the nuclei occur in groups give spectra of rather similar form. The spectra of hydrated crystals provide an example.

The two protons in the water molecule here constitute a pair of nuclear magnets, which are nearest neighbours. The dipole-dipole interaction formally can be divided into two parts one from the interaction between the protons in the water molecule giving a shape function $Q(\xi)$, and one from the interaction with the other neighbours giving a dipole-broadened function $P(\zeta)$.

$Q(\xi)$ for a diamagnetic polycrystalline hydrate is, for example given by Andrew ${ }^{7}$ as

$$
\begin{gathered}
Q(\xi)=Q_{1}(\xi)+Q_{2}(\xi) \\
Q_{1}(\xi)=\frac{1}{4 \sqrt{3} \cdot b} \cdot \frac{1}{\sqrt{1+(\xi / b)}} \text { for }-b<\xi<2 b \\
Q_{2}(\xi)=\frac{1}{4 \sqrt{3} \cdot b} \cdot \frac{1}{\sqrt{1-(\xi / b)}} \text { for }-2 b<\xi<b
\end{gathered}
$$

where $b=(3 / 2) \times\left(\mu / r^{3}\right)$ is the proton moment and $r$ is the distance between the nuclei. 
Here is

$\int_{-\infty}^{+\infty} Q(\xi) \cos u \xi \mathrm{d} \xi=\frac{1}{4 \sqrt{3} \cdot b} \cdot \int_{-b}^{2 b} \frac{\cos u \xi \mathrm{d} \xi}{\sqrt{1+(\xi / b)}}+\frac{1}{4 \sqrt{3} \cdot b} \cdot \int_{-2 b}^{b} \frac{\cos u \xi \mathrm{d} \xi}{\sqrt{1-(\xi / b)}}$

With the substitution $Z=\sqrt{(2 u b / \pi)[1+(\xi / b)]}$ in the first integral and the substitution $Z=\sqrt{(2 u b / \pi)[1-(\xi / b)]}$ in the second integral eqn. (13) is transformed to

$$
\begin{gathered}
\int_{-\infty}^{+\infty} Q(\xi) \cos u \xi \mathrm{d} \xi=\sqrt{\pi / 6 u b} \cdot \cos (u b) \int_{0}^{\sqrt{6 u b / \pi}} \cos \frac{\pi}{2} Z^{2} \mathrm{~d} Z+ \\
+\sqrt{\pi / 6 u b} \sin (u b) \int_{0}^{\sqrt{6 u b / \pi}} \sin \frac{\pi}{2} Z^{2} \mathrm{~d} Z
\end{gathered}
$$

The zeros of the transform of $Q(\xi)$ is shown in Fig. 1. For the calculation of the transform the values of the Fresnel integrals

$$
C\left(\sqrt{6 u b / \pi)}=\int_{0}^{\sqrt{6 u b / \pi}} \cos \frac{\pi}{2} Z^{2} \mathrm{~d} Z \text { and } S(\sqrt{6 u b / \pi})=\int_{\mathrm{o}}^{\sqrt{6 u b / \pi}} \sin \frac{\pi}{2} Z^{2} \mathrm{~d} Z\right.
$$

have been taken from a table given by van Wijngaarden and Scheen ${ }^{8}$.

The first three zeros occur for $u_{1} b=2.16, u_{2} b=5.63$ and $u_{3} b=8.53$ from which $b$ and $r$ can be determined. From the values of the quotients $u_{2} / u_{1}$ and $u_{3} / u_{1}$ it can be tested whether the conditions necessary for a good adaptation are fulfilled.

Spectra composed by separate lines can also be evaluated by means of Fourier transforms.

An absorption line composed by $n$ lines can be written as

$$
f(x)=\sum_{\nu=1}^{n} P_{\nu}\left(x-\xi_{\nu}\right)
$$

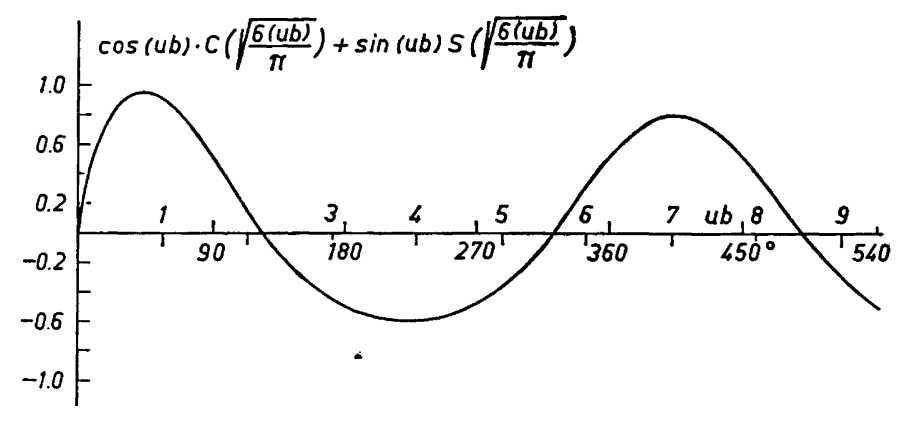

Fig. 1. The function $\cos (u b) \cdot \int_{0}^{\sqrt{6 u b / \pi}} \cos \frac{\pi}{2} Z^{2} \mathrm{~d} Z+\sin (u b) \cdot \int_{0}^{\sqrt{6 u b / \pi}} \sin \frac{\pi}{2} Z^{2} \mathrm{~d} Z$.

Acta Chem. Scand. 16 (1962) No. 9 
The function $P_{\nu}$ is the dipole-function of line number $v$, which is symmetric with its symmetry point for $x=\xi_{v}$.

A spectrum composed by two separate lines can be studied as a simple example.

$$
f(x)=P_{1}\left(x-\xi_{1}\right)+P_{2}\left(x-\xi_{2}\right)
$$

When the lines overlap, it is of importance to determine the value of the displacement $\left(\xi_{2}-\xi_{1}\right)$ and the line-shapes for the two components.

In this case $\xi_{1}$ and $\xi_{2}$ are unknown, and it is convenient to calculate the transforms $F(u, a)$ and $G(u, a)$ for an arbitrary origin.

$$
\begin{aligned}
& F(u, a)=\int_{-\infty}^{+\infty} f(x) \cdot \cos u(x-a) \mathrm{d} x=S_{1}(u) \cdot \cos u\left(\xi_{1}-a\right)+S_{2}(u) \cdot \cos u\left(\xi_{2}-a\right) \\
& G(u, a)=\int_{0}^{+\infty} f(x) \cdot \sin u(x-a) \mathrm{d} x=S_{1}(u) \cdot \sin u\left(\xi_{1}-a\right)+S_{2}(u) \cdot \sin u\left(\xi_{2}-a\right)
\end{aligned}
$$

Where

$$
\begin{aligned}
& S_{1}(u)=\int_{-\infty}^{+\infty} P_{1}(\zeta) \cdot \cos u \zeta \mathrm{d} \zeta, S_{2}(u)=\int_{-\infty}^{+\infty} P_{2}(\zeta) \cdot \cos u \zeta \mathrm{d} \zeta . \\
& \text { If } a=\frac{1}{2} \cdot\left(\xi_{1}+\xi_{2}\right) \text { we get } \\
& F(u, a)=\left\{S_{1}(u)+S_{2}(u)\right\} \cdot \cos \frac{1}{2} \cdot u\left(\xi_{2}-\xi_{1}\right) \\
& G(u, a)=\left\{S_{1}(u)-S_{2}(u)\right\} \cdot \sin \frac{1}{2} \cdot u\left(\xi_{2}-\xi_{1}\right)
\end{aligned}
$$

The zeros of $F(u, a)$ and $G(u, a)$ are studied as functions of $u$ for different $a$-values. By means of iterational calculations that $a$-value is determined for which the zeros of $\cos \frac{1}{2} u\left(\xi_{2}-\xi_{1}\right)$ and $\sin \frac{1}{2} u\left(\xi_{2}-\xi_{1}\right)$ can be identified among the zeros of $F(u, a)$ and $G(u, a)$, respectively.

The displacement $\left(\xi_{2}-\xi_{1}\right)$ is determined from the $u$-values for the zeros of these two series. $S_{1}(u)$ and $S_{2}(u)$ are then easily obtained from eqns. (19) and $(20)$.

In application, the transforms have only to be calculated for a value of $a$, for instance $a=0$, since

$$
\begin{aligned}
& F(u, a)=F(u, 0) \cdot \cos (u a)+G(u, 0) \cdot \sin (u a) \\
& G(u, a)=G(u, 0) \cdot \cos (u a)-F(u, 0) \cdot \sin (u a)
\end{aligned}
$$

A few spectra of this kind have been evaluated. Spectra with a known value of the chemical shift $\left(\xi_{2}-\xi_{1}\right)$ have been gained through numerical addition of single spectral lines. The ${ }^{1} \mathrm{H}$-resonance of water solutions of $\mathrm{MnSO}_{4}$ have been used. The results show that the method is applicable when $\left(\xi_{2}-\xi_{1}\right)$ 
is in the interval $0.5 \mathrm{~m}$ to $3.0 \mathrm{~m}$, where $m$ is the linewidth parameter (for the definition of $m$, cf. Ref. $\left.{ }^{9}\right)$. In this interval $\left(\xi_{2}-\xi_{1}\right)$ has been determined with an error ranging from $\pm 6 \%$ to $\pm 2 \%$ for spectra with good signal/noise-ratio. When $\left(\xi_{2}-\xi_{1}\right)$ is smaller than $0.5 \mathrm{~m}$, the series of zeros in the trigonometric functions cannot be distinguished from those in $\left\{S_{1}(u)+S_{2}(u)\right\}$ and $\left\{S_{1}(u)-S_{2}(u)\right\}$. When $\left(\xi_{2}-\xi_{1}\right)$ is larger than $3.0 \mathrm{~m}$, the displacement can be determined directly from the spectrum.

The ${ }^{1} \mathrm{H}$-resonance of hydrated crystals constitutes a special case of the above mentioned spectral type. A single crystal, where the water molecule has one crystallographic orientation, gives a symmetric spectrum

where

$$
\begin{aligned}
& f(x)=P(x-\xi)+P(x+\xi) \\
& \xi=\frac{3}{2} \mu r^{-3} \cdot\left(3 \cos ^{2} \Theta-1\right)
\end{aligned}
$$

$\mu$ is the proton moment. $r$ is the distance between the nuclei and $\Theta$ is the angle between the magnetic field and the proton-proton vector.

The line-width is rather large compared to the maximum separation between the lines $3 \mu r^{-3}$. Therefore, in a large interval, $\xi$ cannot be directly determined from the spectrum.

With the origin in the symmetry-point of the spectrum we get

$$
F(u)=2 \cdot S(u) \cdot \cos (u \xi), \text { where } S(u)=\int_{-\infty}^{+\infty} P(\zeta) \cdot \cos (u \zeta) \mathrm{d} \zeta
$$

assuming $P$ to be symmetric.

A series of zeros in $F(u)$ thus occur for $u \cdot \xi=\frac{\pi}{2}+n \pi \quad n=0,1,2, \ldots$, from which $\xi$ can be determined. The symmetry properties of $P$ may be ascertained from the values of the quotients of the zeros.

Spectra from single crystals affected by first-order quadrupole interaction are also composed by separate lines. For spin $I$ such a spectrum can be written

$$
f(x)=\sum_{n=k}^{-k} P_{n}(x-n \cdot \xi)
$$

where $k=I-\frac{1}{2}$ and $\xi=v_{Q} f_{o} ; c f$. Cohen and Reif ${ }^{10}$.

The position of the resonance of the unaffected signal can, in these cases, be determined experimentally, and the transforms can be computed with this point as the origin.

For $I=1$ is

$$
\begin{aligned}
& f(x)=P_{1 / 2}\left(x-\frac{\xi}{2}\right)+P_{-1 / 2}\left(x+\frac{\xi}{2}\right) \text { and } \\
& F(u)=\left\{S_{1 / 2}(u)+S_{-1 / 2}(u)\right\} \cdot \cos \left(u \frac{\xi}{2}\right) \\
& G(u)=\left\{S_{1 / 2}(u)-S_{-1 / 2}(u)\right\} \cdot \sin \left(u \frac{\xi}{2}\right)
\end{aligned}
$$

Acta Chem. Scand. 16 (1962) No. 9 
and $\xi$ can be determined from the zeros of $F(u)$ and $G(u)$. If $P_{-1 / 2}$ and $P_{+1 / 2}$ are identcial, the spectrum will be symmetric and can be treated in the same way as the spectrum of single hydrated crystals, described above.

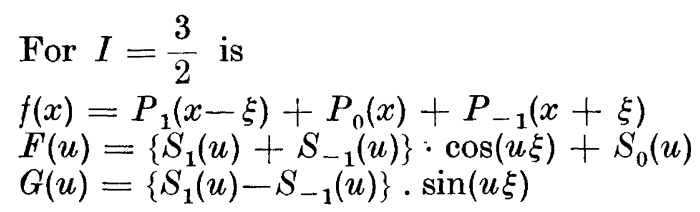

and $\xi$ can here be determined from the zeros of $G(u)$. If the line-shapes of the three lines are identical, the spectrum will be symmetric. The relative intensities of the lines are determined by the quadrupole interaction and in this case is

$$
\begin{aligned}
& S_{0}(u)=\frac{4}{10} \cdot\left\{S_{1}(u)+S_{0}(u)+S_{-1}(u)\right\} \\
& S_{1}(u)=S_{-1}(u)=\frac{3}{10} \cdot\left\{S_{1}(u)+S_{0}(u)+S_{-1}(u)\right\}
\end{aligned}
$$

Thus $\quad F(u)=\left\{\frac{6}{10} \cdot \cos (u \xi)+\frac{4}{10}\right\} \cdot\left\{S_{1}(u)+S_{0}(u)+S_{-1}(u)\right\}$

and $\xi$ can be determined from the series of zeros in $\cos (u \xi)=-\frac{2}{3}$ occurring in $F(u)$.

For $I>\frac{3}{2}$ a similar method may be used if the line-shapes of the different lines are identical.

It seems probable that the evaluation of many spectra can be facilitated by the use of Fourier transforms. No attempt, however, has been made to go into the mathematical side of the problem. The cases, as described above, only serve to examplify some simple practical applications of the method.

Acknowledgements. The author would like to thank the Head of the NMR group Dr. E. Forslind and Dr. U. Hellsten, for their kind interest in this work. The financial support provided by the Swedish Natural Science Research Conncil and the State Council of Technical Research is gratefully acknowledged.

\section{REFERENCES}

1. Andrew, E. R., Bradbury, A. and Eades, R. G. Nature 183 (1959) 1802.

2. Hughes, D. G. and Mac Donald, D. K. C. Proc. Phys. Soc. 78 (1961) 75.

3. Itoh, J., Kusaka, R., Yamagata, Y., Kiriyama, R. and Ibamoto, H. J. Phys. Soc. Japan 8 (1953) 293.

4. Silver, A. H., Kushida, T. and Lambe, J. Phys. Rev. 124 (1962) 1147.

5. Otsuka, E. and Kawamura, H. J. Phys. Soc. Japan 12 (1957) 1071.

6. Kopal, Z. Numerical Analysis, Chapman Hall Ltd. London 1955, p. 408.

7. Andrew, E. R. Nuclear Magnetic Resonance, Cambridge, University Press 1956, p. 155.

8. van Wijngaarden, A. and Scheen, W. L. Nederl. Akad. v. Wetenschappen 19, Amsterdam 1949.

9. Svanson, S. E. and Forslind, E. Acta Chem. Scand. 16 (1962) 2149.

10. Cohen, M. H. and Reif, F. Solide State Phys. 5 (1957) 321.

Received May 2, 1962. 